\title{
Patterning and compartment formation in the diencephalon
}

\section{Mallika Chatterjee and James Y. H. Li*}

Department of Genetics and Developmental Biology, University of Connecticut Health Center, Farmington, CT, USA

Edited by:

Steffen Scholpp, Karlsruhe Institute of

Technology, Germany

\section{Reviewed by:}

Steffen Scholpp, Karlsruhe Institute of

Technology, Germany

Clemens Martin Kiecker, King's

College London, UK

*Correspondence:

James Y. H. Li, Department of

Genetics and Developmental Biology,

University of Connecticut Health

Center, 400 Farmington Avenue,

Farmington, CT 06030, USA.

e-mail: jali@uchc.edu
The diencephalon gives rise to structures that play an important role in connecting the anterior forebrain with the rest of the central nervous system. The thalamus is the major diencephalic derivative that functions as a relay station between the cortex and other lower order sensory systems. Almost two decades ago, neuromeric/prosomeric models were proposed describing the subdivision and potential segmentation of the diencephalon. Unlike the laminar structure of the cortex, the diencephalon is progressively divided into distinct functional compartments consisting principally of thalamus, epithalamus, pretectum, and hypothalamus. Neurons generated within these domains further aggregate to form clusters called nuclei, which form specific structural and functional units. We review the recent advances in understanding the genetic mechanisms that are involved in the patterning and compartment formation of the diencephalon.

Keywords: thalamus, compartment boundary, organizer, fate mapping, ZLI, Gbx2, Shh, prosomere

\section{INTRODUCTION}

Multiple bulges called brain vesicles become visible in the anterior neural tube soon after closure of the neural tube, due to tissue expansion and constriction between the vesicles. The anteriormost vesicles are called the telencephalon and diencephalon, and together they form the future forebrain. The telencephalon gives rise to the neocortex and basal ganglia, while the diencephalon generates structures that connect the neocortex and the forebrain limbic systems with the rest of the central nervous system. The diencephalon is therefore dubbed as the "interbrain" as it functions as a crucial relay and integration center, and modulates sensory, motor, and cognitive functions. Based on histological landmarks and gene expression patterns, the diencephalon is divided into segments called prosomeres, from which major anatomical structures including the pretectum, the habenula, thalamus, prethalamus, and hypothalamus arise. Compartmental development is an evolutionarily conserved mechanism that controls generation of diversity and specificity among different progenitor domains. Furthermore, the compartment boundaries often serve as signaling centers that regulate development of cells in the neighboring compartments. Here, we will review recent studies addressing the patterning and compartment formation of the diencephalon. These studies have identified the signaling molecules that pattern the diencephalon and the intrinsic determinants of different prosomeres and subdivisions of these prosomeres. Recent studies have also demonstrated that there are several lineage restriction boundaries, some of which correspond to the prosomeric borders or subdivisions. These new findings combined with other exciting advances in this field have added to our expanding knowledge of diencephalic development.

\section{PROSOMERE MODEL: DIVISION OF THE DIENCEPHALON}

Morphological studies in chick embryos have suggested that the forebrain and in particular the diencephalon can be divided into multiple transverse segments called neuromeres that are orthogonal to the long axes of the neural tube (Bergquist and Kallen, 1954, 1955; Kaellen, 1965; Vaage, 1969; Puelles et al., 1987). Based on morphology, position of axonal tracts and expression of celladhesion molecules, Figdor and Stern divided the diencephalon into four transverse neuromeres (D1-D4), with the rostral-most D1 adjacent to the telencephalon and D4 abutting the mesencephalon (Figdor and Stern, 1993). In the same year, Bulfone et al. (1993) showed three distinct segments called prosomeres (p1-p3) in the diencephalon by analyzing four developmental regulator genes, Dlx1,Dlx2, Gbx2, and Wnt3. This and subsequent studies have led to the "prosomere model," which divides the developing forebrain into six prosomeres (Puelles and Rubenstein, 1993, 2003; Rubenstein et al., 1994). The p1-p3 segments give rise to the pretectum (p1), the epithalamus and thalamus (previously called the dorsal thalamus) (p2), and prethalamus (p3, previously called ventral thalamus) and eminentia thalami (Figure 1). P1 and p2 correspond to D1 and D2, while p3 may be subdivided into D3 and D4. Although the number and their nature as lineage-restricted compartments have been controversial, the prosomere model provides a conceptual framework for understanding forebrain development by imparting morphological meaning to gene expression patterns, and thus is essential for genetic and comparative studies of the developing brain across species.

\section{ZLI, A COMPARTMENT BOUNDARY OR A SELF-CONTAINED COMPARTMENT?}

Compartmental development is a fundamental mechanism for coordinating growth and patterning of the embryonic field in both invertebrates and vertebrates (Lumsden and Krumlauf, 1996; Kiecker and Lumsden, 2005). Rhombomeres in the vertebrate hindbrain are lineage-restricted compartments, in which cells freely intermingle within the same rhombomere but not with cells 


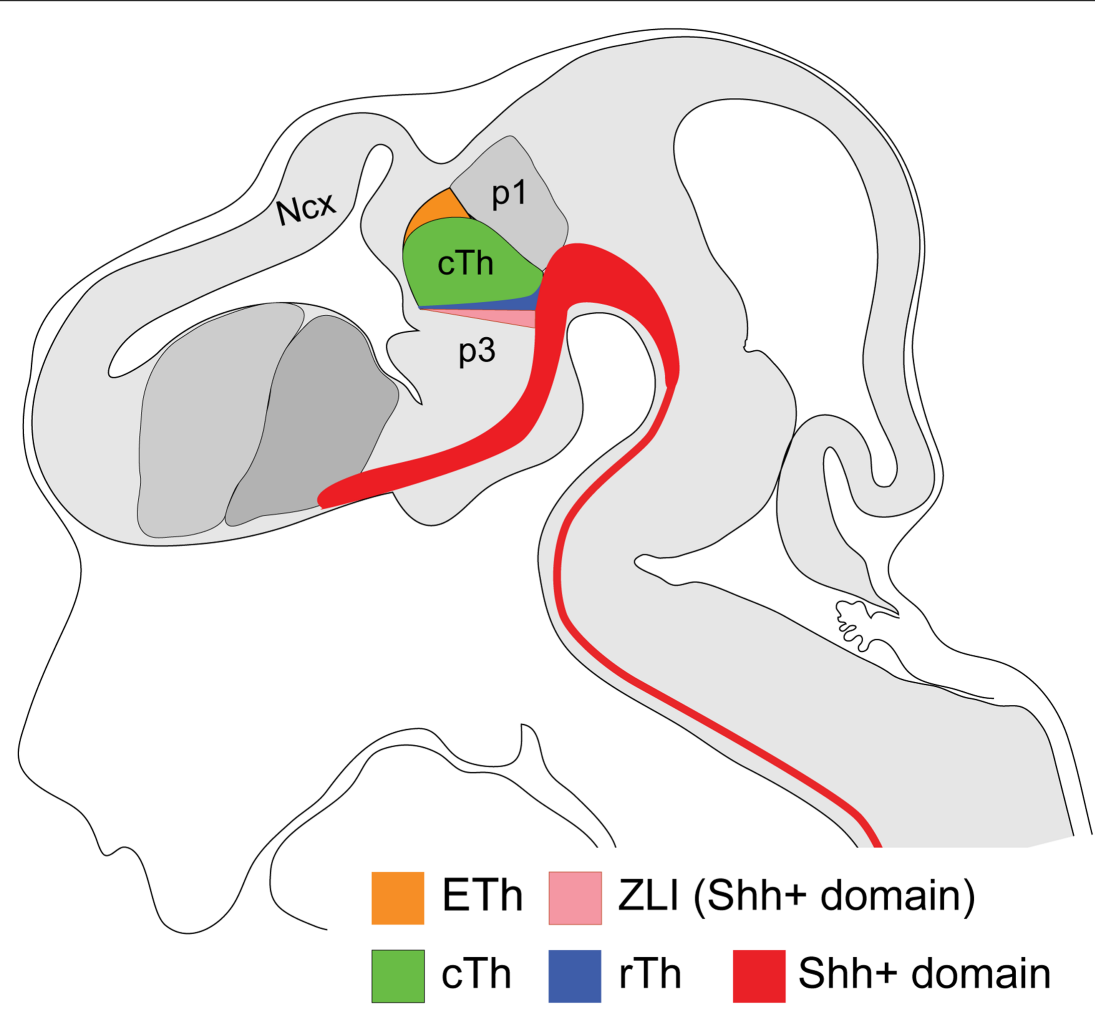

FIGURE 1 | Schematic representation of a sagittal view of the various domains of the diencephalon in mouse embryos at E12.5. The prosomere model predicts that the diencephalon consists of three principal prosomeres/segments. Prosomere1 (p1) corresponds to the pretectum and $\mathrm{p} 3$ corresponds to the prethalamus. The $\mathrm{p} 2$ domain consists of the progenitor domains of the rostral thalamus (rTh), the caudal thalamus (cTh), and the epithalamus. The progeny of the latter give rise to the Gbx2-positive thalamus proper. The ZLI wedge arises from the Shh positive basal plate and acts as an organizer. The entire area shaded in gray indicates the neural tube. Ncx: Neocortex. of the neighboring rhombomeres (Fraser et al., 1990). Each of these rhombomeres displays unique molecular identity and functional organization (Lumsden and Krumlauf, 1996). It is thus interesting to determine whether segmental development occurs in the developing forebrain in a similar fashion. By labeling single or small groups of cells in the diencephalon of chick embryos, Figdor and Stern showed that cells of each neuromere could intermingle freely within the neuromere but were restricted from crossing the border after the formation of morphological ridges at the border (Figdor and Stern, 1993). Compartmental development of the diencephalon is further supported by fate-mapping studies in chick-quail chimera (Garcia-Lopez et al., 2004). However, clonal analyses using retrovirus in chick embryos found broad dispersal of labeled clones without respecting prosomeric boundaries, casting doubts on the compartmental development within the diencephalon (Arnold-Aldea and Cepko, 1996; Golden and Cepko, 1996). Furthermore, Larsen et al. (2001) showed that there was no obvious cell segregation at the border between $\mathrm{p} 1$ and p2 in chick embryos. However, they observed lineage segregation between p1 and the mesencephalon, as well as a lineage boundary between p 2 and p 3 (Larsen et al., 2001). Wedged in between p 2 and p3 is a transverse domain called the zona limitans intrathalamica (ZLI), which is defined by the expression of Sonic hedgehog (Shh; Zeltser et al., 2001; Figure 1). Fate-mapping studies showed that
ZLI cells were segregated from those of p2 or p3 cells (Zeltser et al., 2001). The author thus suggested that the ZLI is a self-contained compartment (Zeltser et al., 2001), rather than a compartment boundary (Garcia-Lopez et al., 2004). During embryogenesis, the prospective ZLI is first defined by the absence of expression of Lfng (Zeltser et al., 2001), which encodes a glycosyltransferase that modulates Notch signaling. Its homolog plays an important role in compartment boundary formation in Drosophila (Cho and Choi, 1998; Dominguez and de Celis, 1998; Papayannopoulos et al., 1998; Rauskolb et al., 1999). Perturbation of Lfng function disrupted formation of the compartment boundaries flanking the ZLI in chick embryos (Zeltser et al., 2001), suggesting that Lfng-mediated cell sorting contributes to the establishment of the ZLI compartment. Similar to the chick embryo, the prospective ZLI is also defined as a negative expression domain of $L f n g$ and its paralog $M f n g$ in mice (Zeltser et al., 2001; Baek et al., 2006). However, no developmental defect in the neural tube lacking $L f n g$ has been reported so far in mice (Zhang and Gridley, 1998). Further studies are needed to determine whether Lfng and/or Mfng play a similar role in regulating ZLI formation in mice.

Notch signaling is known to play an important role in the formation of compartment boundaries in various systems (Cheng et al., 2004; Tossell et al., 2011a,b). Interestingly, a Notch effector gene, Hes1, is expressed in the boundary regions of the neural tube, 
such as the isthmus, ZLI, and rhombomeric borders (Baek et al., 2006). Hes proteins inhibit neurogenesis and promote gliogenesis (Kageyama et al., 2008). Generation of neuron-free zones is associated with the formation of specialized cells, called boundary cells, at the border of the compartments (Baek et al., 2006; Jukkola et al., 2006). Importantly, various compartment boundaries, including the ZLI, were missing in mouse embryos lacking both Hes1 and its related gene Hes5 (Baek et al., 2006). Similarly, knock-down of her6 (equivalent to mammalian Hes1) leads to loss of the organizer (Scholpp et al., 2009). These observations demonstrate the essential role of Hes genes in the formation of these compartment boundaries. As other signaling pathways such as FGF and Shh have been shown to directly regulate Hes1 expression (Ingram et al., 2008; Wall et al., 2009; Sato et al., 2010), it remains to be determined how Notch interacts with other signaling pathway to regulate Hes genes in the formation of the ZLI compartment. Therefore, studying regulation and function of Hes genes may provide insights into the establishment of the ZLI compartment.

\section{THE FORMATION AND FUNCTION OF THE ZLI ORGANIZER}

Compartment boundaries often serve as a signaling center, also called an organizer, to regulate cell fate specification of progenitors in the neighboring compartments (Irvine and Rauskolb, 2001; Kiecker and Lumsden, 2005). For example, the isthmic organizer at the midbrain-hindbrain junction patterns the developing midbrain and cerebellum through Fgf8 signaling (Wurst and Bally-Cuif, 2001; Sato et al., 2004). Genetic fate-mapping studies have demonstrated that the midbrain-hindbrain border is a lineage restriction boundary (Zervas et al., 2004; Langenberg and Brand, 2005; Sunmonu et al., 2011). The ZLI expresses multiple signaling molecules including Shh, and members of the Wnt and FGF families (Echevarria et al., 2003). Transplantation and genetic manipulation experiments have demonstrated that the ZLI acts as an organizing center and Shh is the main component of the ZLI organizer activity (Hashimoto-Torii et al., 2003; Kiecker and Lumsden, 2004; Vieira et al., 2005; Scholpp et al., 2006). In thalamic explants, different concentrations of Shh proteins induced differential expression of Sox14 and Gbx2, supporting the notion that Shh proteins secreted from the ZLI act as a morphogen to control the pattern formation of the $\mathrm{p} 2$ domain (Hashimoto-Torii et al., 2003).

Based on development of the organizer in different systems, Meinhardt has proposed that the formation of an organizing center involves initial specification of two populations of cells in adjacent territories and subsequent induction of cells at the common border to express signaling molecules (Meinhardt, 1983). Grafting and co-culture experiments indeed demonstrated that juxtaposition of prethalamic and thalamic tissues was sufficient to induce Shh expression at the interface (Vieira et al., 2005; Guinazu et al., 2007). Several studies have revealed the molecular basis for positioning the prospective ZLI and specification of the prethalamus and the thalamus in chicken (Kobayashi et al., 2002; Braun et al., 2003; Echevarria et al., 2003; Vieira et al., 2005), zebrafish (Scholpp et al., 2006), Xenopus (Rodriguez-Seguel et al., 2009), and mice (Hirata et al., 2006). It was proposed that mutual repression between Six3 and Irx3 positioned the prospective ZLI in chick embryos (Kobayashi et al., 2002; Braun et al., 2003). However, the ZLI was present in Six3-deficient mice, indicating that Six3 is not essential for ZLI formation (Lavado et al., 2008). Zinc-finger genes Fezf1 and Fezf2 are expressed in the rostral forebrain juxtaposed with the rostral limit of Irx 1 expression in mouse embryos (Hirata et al., 2004, 2006). Deletion of Fezf1 and Fezf2 in mice or only fezf 2 in fish disrupts formation of ZLI and abnormal expression of genes characteristic for the thalamus or pretectum in the prethalamus (Hirata et al., 2006; Jeong et al., 2007). These results demonstrate that Fezf1 and Fezf2 are important for the formation of the prethalamus and the ZLI. However, deleting Fezf1 and Fezf2 did not completely abolish the induction of the prethalamic territory. Furthermore, a fate-mapping study in zebrafish revealed that the fate of the prethalamus was established during gastrulation (Staudt and Houart, 2007). These results suggest that other factors in addition to Fezf1 and Fezf 2 may be involved in the induction of the prethalamus.

In mouse embryos, the expression domain of Fezf1 and Fezf2 abuts that of Irx 1 (Hirata et al., 2006). Furthermore, genetic studies showed that mutual repression between Fezf and Irx genes positioned the prospective ZLI in Xenopus (Rodriguez-Seguel et al., 2009) and mouse embryos (Hirata et al., 2006). In mice, there are six Irx genes in two genomic clusters, and genes of the same cluster have similar expression pattern in the diencephalon (Peters et al., 2000; Houweling et al., 2001; Gomez-Skarmeta and Modolell, 2002). The potential redundancy and close linkage of different Irx genes in the mouse genome create difficulties to uncover their function by gene targeting knock-out experiments (Peters et al., 2002; Anselme et al., 2007). The best available tool to analyze Irx function in mouse so far is the naturally occurring Fused toes mutant, which shows a fuzzier and reduced expression of Shh in the ZLI and the basal plate at E9.5, suggesting that the ZLI formation is probably affected due to loss of the $\operatorname{Ir} x B$ cluster (Anselme et al., 2007). However, the interpretation is complicated by the simultaneous loss of three other additional genes (Fts, Ftm, and Fto) of unknown function in the nervous system (Peters et al., 2002).

In zebrafish, the expression domains of fezf and irx $1 b$ (irx7 as well) do not juxtapose with each other (Scholpp et al., 2007). Furthermore, knock-down of irx $1 b$ resulted in a caudal expansion of the ZLI, rather than a loss of the ZLI (Scholpp et al., 2007). During gastrulation, members of Otx family, Otx1 and Otx2, are broadly expressed in the prospective forebrain and midbrain (Simeone et al., 1992). In zebrafish embryos, otx2 is down-regulated in the anterior neural tube and only maintained in a region posterior to the prethalamus, including the ZLI and thalamus, at the 10somite stage (Scholpp et al., 2007). Knock-down of otx1 and otx2 proteins immediately before ZLI formation prevented expression of ZLI markers, including shh, and conditional expression of ot $x 2$ cell-autonomously rescued shh expression in the prospective ZLI (Scholpp et al., 2007). Based on these observations, Scholpp et al. have proposed that the positioning and induction of ZLI is determined by the fezf-otx interface, while fezf and irx $1 b$ define the anterior and posterior limits of ZLI domain, respectively (Scholpp et al., 2007; Scholpp and Lumsden, 2010). However, strong Otx2 expression was maintained in the prethalamus and ZLI in mouse embryos at least until E12.5 after the formation of the ZLI at E10.5 (Chatterjee and Li, unpublished observations). 
Furthermore, loss-of-function studies have shown that both Otx1 and $O t \times 2$ are required, in a dosage dependent manner, for the formation of both diencephalon and mesencephalon, including the prethalamus, ZLI, and thalamus, in mice (Acampora et al., 1997; Kurokawa et al., 2004; Puelles et al., 2006; Sakurai et al., 2010). Therefore, future studies are necessary to determine the mechanism underlying the positioning and induction of ZLI in mouse embryos.

Shh expression expands from the basal plate dorsally into the wedge-shaped ZLI. Cell fate mapping have shown that the characteristic progression of Shh expression is not a result of ventral-todorsal cell movement (Zeltser, 2005; Scholpp et al., 2006; Staudt and Houart, 2007). Therefore, Shh expression in the ZLI is probably induced by a polarized signaling in the ventral-dorsal direction (Zeltser, 2005). Using chick forebrain explant culture, Zeltser showed that Shh signaling from the basal plate was required for ZLI Shh expression in the alar plate and the dorsal progression of the ZLI organizer is regulated by inhibitory signals from the dorsal diencephalon (Zeltser, 2005). However, using a similar approach, Guinazu et al. (2007) demonstrated that Shh expression in the basal plate was dispensable for the induction of the ZLI. The latter result is supported by genetic studies in zebrafish and mice (Scholpp et al., 2006; Jeong et al., 2011). In zebrafish, the ZLI shh expression was present in one-eyed pinhead mutants, which lacked the axial mesoderm and basal plate shh expression (Scholpp et al., 2006). By deleting a 525-bp intronic sequence upstream of Shh coding region called Sh Brain Enhancer-1 (SBE-1), Jeong et al. (2011) specifically abolished Shh expression and activity in the basal plate of the mouse forebrain. Normal expression of Shh and other ZLI markers like Sim1 and Sim2 was found in the ZLI of these SBE-1 deletion mice (Jeong et al., 2011). Altogether, these observations indicate that Shh expression from the basal plate is not crucial for ZLI development in vertebrates. A recent report have shown that the requirement of $B$-catenin/Wnt signaling in the formation of the ZLI organizer in the zebrafish (Mattes et al., 2012). Blocking B-catenin signaling or knocking down of both wnt3 and wnt $3 a$ mostly prevented ZLI organizer formation within a narrow time window of 10-14 h post-fertilization in zebrafish. Interestingly, the loss of ZLI shh expression was associated with enhanced apoptosis of the organizer cells and inhibition of apoptosis by simultaneous knock-down of p53 rescued the ZLI organizer in the absence of wnt3/wnt3a. These observations suggest that $ß$-catenin/Wnt signaling is mainly required for the survival of ZLI organizer cells. The molecular nature of signals that directly induce formation of the ZLI organizer remains to be determined.

In the aforementioned study, Guinazu et al. (2007) also showed that signals from the dorsal diencephalon antagonized ZLI formation, and they suggested that retinoic acid (RA) is a dorsal ZLI inhibitor. Indeed, Cyp1b1, which encode cytochrome p450 family of mono-oxygenases to promote RA synthesis, is expressed in the chick epithalamus (Chambers et al., 2007; Guinazu et al., 2007). Furthermore, in ovo electroporation experiments showed that Cyp1b1 acted cell-non-autonomously to inhibit Shh expression in the prospective ZLI, in agreement with the involvement of a diffusible molecule like RA (Guinazu et al., 2007). However, no brain defect has been reported in Cyp1b1-null mice (Buters et al., 1999; Libby et al., 2003).

\section{SUBDIVISION AND COMPARTMENTAL DEVELOPMENT WITHIN THE P2 DOMAIN}

The major p2-derived structure is the thalamus, which functions as a relay station and integration center for almost all sensory and motor information to and from the cortex (Jones, 2007). However, in addition to the thalamus, the $\mathrm{p} 2$ domain is further subdivided to give rise to the epithalamus, located dorsal to the thalamus (Rubenstein et al., 1994). Gene expression and short-term lineage tracing experiments have recently revealed that the thalamic progenitor domain can be further divided into rostral and caudal areas (Figure 1). The caudal thalamus (cTh) gives rise to glutamatergic neurons, which project to the cortex, and cell bodies of these neurons constitute the nuclear complex that is traditionally viewed as the thalamus (Jones, 2007; Vue et al., 2007; Chen et al., 2009). In contrast, the rostral thalamus ( $\mathrm{rTh}$ ) produces GABAergic neurons, which do not project to the cortex (Vue et al., 2007). Significant progress has been made in the study of the development and compartmentalization of the $\mathrm{p} 2$ domain. The rest of the review will focus on patterning and compartment formation within the $\mathrm{p} 2$ domain.

\section{ZLI ORGANIZER SIGNALS DETERMINE THE POSITION OF THE BORDER BETWEEN THE rTh AND cTh}

By analyzing an array of transcription factors that are known for their important roles in regulating cell fate decision, Vue et al. (2007) identified the rostral thalamic progenitor domain (rTh), which is located immediately caudal to the ZLI. The rTh

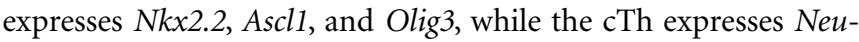
rog1, Neurog2, and Olig3 (Vue et al., 2007). Moreover, Kataoka and Shimogori showed that the rTh, also called the Rim, is a heterogeneous structure containing distinct populations of cells that express Tal2, Six3/Gad67, Nkx2.2/Sox14, and Arx, respectively (Kataoka and Shimogori, 2008). Vue et al. (2009) demonstrated that enhancing or attenuating Shh signaling led to the enlargement or reduction of the rTh domain, demonstrating that Shh is important for positioning the border between the rTh and cTh. Significantly, analyzing mouse mutants lacking SBE-1 showed that, although the Shh expression in the ZLI was normal, rTh cells were mis-specified, indicating that the specification of the rTh is dependent on Shh signaling from both the ventral midline and the ZLI (Jeong et al., 2011). Fgf8, a secreted morphogen of the Fgf superfamily, is expressed in the dorsal diencephalon (Crossley et al., 2001; Kataoka and Shimogori, 2008; Martinez-Ferre and Martinez, 2009). Manipulations of Fgf8 signaling by in utero electroporation of Fgf8 or sFGFR3, encoding a soluble form of FGFR3 that blocks most FGF, including Fgf8, activity, resulted in respective enlargement or reduction of the $\mathrm{rTh}$ and its derived nuclei (Kataoka and Shimogori, 2008). Changing Fgf8 activity did not affect Shh and Wnt signaling (Kataoka and Shimogori, 2008). Neither did changing Shh signaling affect the expression of $F g f 8$ and its downstream effectors (Vue et al., 2009). These results suggest that the Shh and Fgf8 signaling pathways converge in patterning the p2 domain via independent mechanisms. Experiments in zebrafish have recently shown that her6, a homolog of Hes1, is important for defining the rTh (Scholpp et al., 2009). her6 is initially expressed throughout $\mathrm{p} 2$, and gradually restricted to the rTh as neurogenesis progresses and neurog1 and neurog 2 expression is induced in $\mathrm{CTh}$ 
cells. Furthermore, her6 is required and sufficient to suppress neu$\operatorname{rog} 1 / 2$ and to induce ascl1. Given their known function in directly regulating Hes1 (Ingram et al., 2008; Wall et al., 2009; Sato et al., 2010), Shh and Fgf8 may independently regulate the formation of the rTh via Hes 1 .

Short-term or long-term lineage tracing using Ascl1-EGFP or Tal1-creER transgenic mouse lines have demonstrated that the rTh cells give rise to GABAergic neurons of the ventral lateral geniculate nucleus (vLG) and intergeniculate leaflet (IGL; Vue et al., 2007; Jeong et al., 2011). Traditionally, the vLG and IGL are considered structures derived from the prethalamus, because they display clear differences from thalamic nuclei in neurochemistry and connectivity (Jones, 2007). Indeed, fate-mapping studies showed that in addition to other prethalamic nuclei, Dlx5/6-expressing cells in the prethalamus contributed to the vLG, demonstrating that the vLG is composed of heterogeneous neurons originating from the rTh and the prethalamus (Jeong et al., 2011). These findings imply that the compartment boundaries between p2 and the ZLI, as well as between $\mathrm{p} 3$ and ZLI mainly restrict the movement of progenitor cells, but not postmitotic cells. Similarly, different restrictions on progenitor cells versus postmitotic cells were previously found at the rhombomeric or pallial-subpallial boundaries (Fishell et al., 1993; Wingate and Lumsden, 1996). Therefore, compartmental boundaries are mainly required for a proliferating cell population with labile cell fates. As the fate of postmitotic cells become specified, boundary restriction becomes dispensable (Kiecker and Lumsden, 2005).

\section{THE cTh IS A SELF-CONTAINED COMPARTMENT}

The cTh is also defined by the expression domain of homeobox gene $G b x 2$ (Bulfone et al., 1993). The onset of Gbx2 expression appears to be associated with cell cycle exit of thalamic neurons (Bulfone et al., 1993; Nakagawa and O'Leary, 2001; Chen et al., 2009). The importance of Gbx2 in thalamic development is demonstrated by the fact that deleting $G b \times 2$ disrupts the histogenesis of the thalamus and abolishes almost the entire thalamocortical projections in mice (Miyashita-Lin et al., 1999; Hevner et al., 2002). Furthermore, $G b \times 2$ is required for the survival of thalamic neurons (Szabo et al., 2009). Using a Gbx2-creER(T2) knock-in mouse line, we performed inducible genetic fate-mapping studies where we showed that the entire thalamic complex was derived from the Gbx2-lineage, and the fate-mapped Gbx2-lineage formed sharp boundaries surrounding the thalamus (Chen et al., 2009). Interestingly, Gbx2-lineage did not contribute to the vLG, while fate-mapping studies using Tal1-creER or Nkx2.2-cre showed that rTh-derived cells mostly contributed to the vLG but not cThderived nuclei, demonstrating that cells derived from the rTh and cTh do not intermingle during development (Chen et al., 2009; Jeong et al., 2011). In the absence of Gbx2, the Gbx2-lineage abnormally contributed to the habenula and pretectum (Chen et al., 2009). Interestingly, although the dorsal and caudal borders of the thalamus were disrupted in Gbx2-deficient mice, the anterior and ventral borders appeared unaffected by the loss of Gbx2, demonstrating that different mechanisms may be employed in establishing these thalamic boundaries. We suggest that the disruption of the boundaries is not caused by mis-specification of the thalamic neurons. In fact, in chimeric or genetic mosaic embryos that were composed of wildtype and Gbx2-deficient cells, the thalamic boundaries were mostly restored, demonstrating that $G b \times 2$ has a cell-non-autonomous role in regulating the formation of thalamic boundaries (Chen et al., 2009).

The disruption of the thalamic boundaries caused by loss of Gbx2 suggests that the establishment of the lineage boundaries surrounding the thalamus is likely achieved through active cell sorting at the border rather than by a general lack of movement of the fate-mapped cells (Chen et al., 2009). It is conceivable that $G b \times 2$ regulates expression of cell-adhesion molecules that in turn control cell segregation between the thalamus and the pretectum. In agreement with the fate-mapping result in mouse embryos, lineage restriction was discovered at the border between thalamus and pretectum in zebrafish embryos (Peukert et al., 2011). $p c d h 10 b$, which encodes a cell-adhesion molecule protocadherin, is expressed in thalamic progenitors with its caudal expression border in register with the thalamic-pretectal border (Peukert et al., 2011). Morpholino mediated knock-down of $p c d h 10 b$ disrupted the lineage restriction boundary between the thalamus and pretectum (Peukert et al., 2011). Significantly, expression of pcdh10b is regulated by LIM-homeodomain (HD) transcription factors lhx 2 and lhx9 (Peukert et al., 2011). We have recently demonstrated that Gbx2 is essential for the normal function of $\operatorname{Lh} \times 2$ and $L h \times 9$ in mice (Chatterjee and Li, submitted). These findings suggest that Gbx2, $L h \times 2$, and $L h x 9$ may work in the same pathway to regulate the expression of $P c d h 10 b$, which in turn controls the compartment boundary between the thalamus and pretectum. As suggested by the authors, it remains to be tested whether Pcdh10b is the celladhesion molecule whose deregulation causes the loss of thalamic boundaries observed in Gbx2-deficient embryos.

\section{DEVELOPMENT OF THE HABENULA}

The p2 domain can be further subdivided into the epithalamus and the thalamus. The epithalamus gives rise to the habenula and pineal gland, which are evolutionarily conserved structures and are present in virtually all vertebrates. In recent years, there is a resurging interest in the habenula because of its regulatory roles in emotive decision-making and its implications in psychiatric disorders (Hikosaka, 2010). The habenula is further divided into the medial and the lateral habenula. The habenula receives input from the cortex, limbic system and basal ganglia through the stria medullaris (Sutherland, 1982; Hikosaka et al., 2008). The fasciculus retroflexus, also known as the habenular-interpeduncular tract, forms the output tract of the habenula and connects with the dopaminergic and serotonergic nuclei in the midbrain and hindbrain. The connectivity of the habenula suggests that it acts as an important node linking the forebrain to the midbrain and hindbrain monoamine systems that are involved in modulating emotional behaviors (Hikosaka, 2010). Indeed, functional imaging studies revealed that the habenula was hyperactive in patients with major depression and in healthy people when receiving negative feedback regarding a failed performance (Morris et al., 1999; Ullsperger and von Cramon, 2003; Hikosaka, 2010).

The habenula has been extensively studied in lower vertebrates like fish, amphibians, and reptile for its remarkable asymmetry in morphology, connectivity, and gene expression (Concha and Wilson, 2001; Halpern et al., 2003; Aizawa et al., 2005; Gamse et al., 
2005). However, relatively little is known about the specification and differentiation of the habenula in mammals. The habenula was once considered to be anatomically continuous with the pretectum (Rose, 1949), and this structure is often overlooked or mis-labeled in literature. There are currently no specific markers for the progenitor domain of the habenula, and the epithalamus shares expression of many marker genes with the pretectum or the thalamus. By contrast, postmitotic neurons of the habenula display distinct molecular markers (Quina et al., 2009). POU-domain homeobox gene Pou4f1 (also known as Brn3a) is expressed in the developing habenula and is essential for habenular development (Xiang et al., 1996; Quina et al., 2009). By comparing the expression profile of the E16.5 habenula with other brain regions or between wildtype and Pou4f1-deficient habenula, Quina et al. (2009) have identified genes that are specifically expressed in postmitotic habenular neurons, including Nurr1, a downstream target of Pou4f1. The authors showed that despite of being highly heterogeneous in its composition, the habenula by itself has a molecular identity that is distinct from other brain regions including the neighboring thalamus. Genetic studies have identified molecules that are important for the development of the habenular neuronal traits, such as axon fasciculation and targeting (Giger et al., 2000; Kantor et al., 2004; Quina et al., 2009). However, how the habenular identity is specified remains unresolved.

Genetic fate-mapping studies have shown that the Gbx2-lineage is mostly restricted from crossing the border between the epithalamus and thalamus (Chen et al., 2009). By contrast, transplantation studies showed that cells derived from epithalamic grafts contributed to the mantle zone of the thalamus in chick embryos (Garcia-Lopez et al., 2004), or in organotypic explants of mouse diencephalon (Martinez-Ferre and Martinez, 2009). There results suggest that by some unknown mechanism the border between the epithalamus and thalamus mainly restrict cell movements in the unilateral direction. Long-term fate-mapping studies are necessary to determine the contribution and function of epithalamus-derived cells in the thalamus.

\section{SIGNALING PATHWAYS THAT CONTROL DIFFERENTIATION OF THE cTh AND THE EPITHALAMUS}

It has been shown that when the entire p2 domain is forced to express constitutively active Smoothened after E10.5 in mouse embryos, the cTh and the epithalamus persist despite the caudal expansion of the rTh (Vue et al., 2009). This suggests that additional signaling mechanisms are involved in controlling the identity of the cTh and epithalamus (Bluske et al., 2009). Recent studies have shown that Wnt and Fgf signaling play a role in development of the thalamus and the epithalamus.

Wnt signaling is important for partitioning of the anterior neural tube into the telencephalon and diencephalon with high Wnt activity inducing diencephalon while inhibition of Wnt promoting telencephalon (Heisenberg et al., 2001; Houart et al., 2002). Using explant culture and in ovo electroporation, Braun et al. (2003) demonstrated that Wnt activity sets up the difference in competence of rostral and caudal forebrain tissues by inducing differential expression Irx3 and Six3 in these two domains. Furthermore, manipulation of Wnt activity demonstrated that Wnt signaling played a key role in determining the position and angle of the ZLI relative to the longitudinal axis of the neural tube, probably by regulating expression of Irx genes (Sylvester et al., 2010). In addition to its patterning role in progenitors, Wnt signaling may be also important for maintaining distinct characteristics in different postmitotic neurons derived from different prosomeres or subdivision of a prosomere. For example, it was shown that Wnt activity is required for maintaining $G b \times 2$ and $D l \times 2$ expression in tissues that express Irx3 and Six3 respectively (Braun et al., 2003). Inactivation of Wnt receptor Lrp6 results in caudalization of pland p2, along with ectopic expression of prethalamic markers in the thalamus and failure of ZLI formation (Zhou et al., 2004). At later stages, Wnt signaling continues to play a role in the differentiation of thalamic nuclei (Liu et al., 2008), and the development of thalamocortical projections (Wang et al., 2002; Zhou et al., 2008 , 2009). Multiple Wnt ligands, as well as components of the Wnt signaling pathway, including receptors (Fz1/2/4/7/8/9/10), inhibitors (Sfrp1/2/3 and Axin1/2), and effectors (Tcf1 and Tcf4), are expressed in discrete domains of the diencephalon (Zhou et al., 2004; Bluske et al., 2009; Quinlan et al., 2009). One remaining challenge is to determine the identity and the mechanism of Wnt ligands in regulating development of the diencephalon.

Recent studies have revealed that FGF signaling is also important for the development of the diencephalon, although its role is less understood. Fgf15 (or Fgf19 in chick or fish embryos) is expressed in the thalamus, and mediates Shh function in cell proliferation within the diencephalon at the early stages in mouse embryos (Ishibashi and McMahon, 2002; Miyake et al., 2005; Gimeno and Martinez, 2007). Inactivation of $f g f 3$ and $f g f 8$ disrupted formation of the prethalamus in zebrafish (Walshe and Mason, 2003). Fgf8 is expressed in the dorsal midline of the diencephalon. Forced expression of Fgf8 by in utero electroporation promotes rTh identity in a Shh-independent pathway (Kataoka and Shimogori, 2008). By analyzing an Fgf8 hypomorphic allele, Martinez-Ferre et al. showed that attenuating Fgf8 caused reduction of the habenula and thalamus (Martinez-Ferre and Martinez, 2009). The authors also showed an expansion of Wnt1 expression in the thalamic midline and loss of Wnt3a expression in Fgf8 mutants. It is possible that the phenotype observed in the habenula in this instance is mediated by Wnt signaling and needs to be verified by additional studies.

\section{CONCLUSIONS AND PERSPECTIVES}

Genetic studies have clearly demonstrated that the ZLI organizer, or specifically Shh signaling, plays an important role of patterning the diencephalon. However, the molecular mechanisms that underlie the establishment of the ZLI or regulate Shh expression is not completely clear. Mutual repression between region-specific genes has emerged as a common mechanism in establishing boundaries, including the ZLI, within the vertebrate neural tube. Previous studies suggest that the ZLI is induced at different interfaces, such as Six3/Irx3 in chicken (Kobayashi et al., 2002; Braun et al., 2003), fezf/otx in zebrafish (Scholpp et al., 2007), and Fezf/Irx in mice (Hirata et al., 2006). Additional studies are required to resolve whether these apparent differences are truly specific to different species, or they reflect differences in the temporal dynamics of gene expression in different species. The timing of the establishment of the ZLI organizer appears different in various species. For 
example, $w n t 8 b$ and $s h$, two ZLI organizer markers, are induced in a transverse band of cells corresponding to the prospective organizer during early and late somitogenesis in zebrafish (Scholpp et al., 2009), whereas the ZLI Shh expression is not formed until much later at E10.5 in mice. It will be interesting to determine how such differences contribute to the evolution of the forebrain. It has been suggested that the relative size of the telencephalon versus diencephalon among ecological variants of the same species of cichlid fishes can be determined by creating different angles of the ZLI structure (Sylvester et al., 2010). This suggests that even though the same genetic mechanism might initially determine the position of the ZLI, further variation can be achieved to allow better adaptation to the external world.

It has been previously shown the compartment boundary mainly restricts cell movements of proliferating progenitors but not postmitotic cells (Fishell et al., 1993; Wingate and Lumsden, 1996; Kiecker and Lumsden, 2005). Lineage-restricted boundaries that were recently revealed by fate-mapping studies in mice seem to also apply to postmitotic cells (Chen et al., 2009; Jeong et al., 2011). Unlike the cortex, where neurons are arranged in laminar structures, diencephalic neurons aggregate to form distinct nuclei. The molecular mechanism that regulates specific grouping of neurons during differentiation of nuclei remains largely

\section{REFERENCES}

Acampora, D., Avantaggiato, V., Tuorto, F., and Simeone, A. (1997). Genetic control of brain morphogenesis through Otx gene dosage requirement. Development 124, 3639-3650.

Aizawa, H., Bianco, I. H., Hamaoka, T., Miyashita, T., Uemura, O., Concha, M. L., Russell, C., Wilson, S. W., and Okamoto, H. (2005). Laterotopic representation of left-right information onto the dorso-ventral axis of a zebrafish midbrain target nucleus. Curr. Biol. 15, 238-243.

Anselme, I., Laclef, C., Lanaud, M., Ruther, U., and SchneiderMaunoury, S. (2007). Defects in brain patterning and head morphogenesis in the mouse mutant fused toes. Dev. Biol. 304, 208-220.

Arnold-Aldea, S. A., and Cepko, C. L. (1996). Dispersion patterns of clonally related cells during development of the hypothalamus. Dev. Biol. 173, 148-161.

Baek, J. H., Hatakeyama, J., Sakamoto, S., Ohtsuka, T., and Kageyama, R. (2006). Persistent and high levels of Hes 1 expression regulate boundary formation in the developing central nervous system. Development 133, 2467-2476.

Bergquist, H., and Kallen, B. (1954). Notes on the early histogenesis and morphogenesis of the central nervous system in vertebrates. J. Comp. Neurol. 100, 627-659.

Bergquist, H., and Kallen, B. (1955). The archencephalic neuromery in ambystoma punctatum; an experimental study. Acta Anat. (Basel) 24, 208-214.

Bluske, K. K., Kawakami, Y., KoyanoNakagawa, N., and Nakagawa, Y. (2009). Differential activity of Wnt/beta-catenin signaling in the embryonic mouse thalamus. Dev. Dyn. 238, 3297-3309.

Braun, M. M., Etheridge, A., Bernard, A., Robertson, C. P., and Roelink, H. (2003). Wnt signaling is required at distinct stages of development for the induction of the posterior forebrain. Development 130, 5579-5587.

Bulfone, A., Puelles, L., Porteus, M. H., Frohman, M. A., Martin, G. R., and Rubenstein, J. L. (1993). Spatially restricted expression of Dlx-1, Dlx2 (Tes-1), Gbx-2, and Wnt- 3 in the embryonic day 12.5 mouse forebrain defines potential transverse and longitudinal segmental boundaries. J.

Buters, J. T., Sakai, S., Richter, T., Pineau, T., Alexander, D. L., Savas, U., Doehmer, J., Ward, J. M., Jefcoate, C. R., and Gonzalez, F. J. (1999). Cytochrome P450 CYP1B1 determines susceptibility to 7, 12-dimethylbenz[a] anthraceneinduced lymphomas. Proc. Natl. Acad. Sci. U.S.A. 96, 1977-1982.

Chambers, D., Wilson, L., Maden, M., and Lumsden, A. (2007). RALDHindependent generation of retinoic acid during vertebrate embryogenesis by CYP1B1. Development 134, 1369-1383. Neurosci. 13, 3155-3172. unknown. In the developing central neural tube, the expression of several cadherin molecules is restricted to developmental compartments as well as nuclei (Redies and Takeichi, 1996; Redies et al., 2000; Yoon et al., 2000). Therefore, establishment of compartments in the diencephalon and nuclei may share common mechanisms. Interestingly, genetic inducible fate mapping of $G b \times 2$ expressing cells at different stages have revealed that different thalamic nuclei display a distinct onset and duration of $G b \times 2$ expression. These observations raise an interesting possibility that the dynamic and differential expression of $G b \times 2$ may lead to segregation of $G b \times 2$-positive neurons from $G b \times 2$-negative neurons, which have not yet started to express $G b \times 2$ or have lost $G b \times 2$ expression.

As we progress in terms of our knowledge in this field, many of the questions raised will be answered and many more interesting will come up. We have not yet reached the threshold of knowing about the diencephalon. So we hope that this excitement will continue.

\section{ACKNOWLEDGMENTS}

This work was partially supported by grants from the National Institute of Health (R01HD050474) and Connecticut Stem Cell program (10SCB30) to James Y. H. Li.
Chen, L., Guo, Q., and Li, J. Y. (2009). Transcription factor Gbx2 acts cell-nonautonomously to regulate the formation of lineagerestriction boundaries of the thalamus. Development 136, 1317-1326.

Cheng, Y. C., Amoyel, M., Qiu, X., Jiang, Y. J., Xu, Q., and Wilkinson, D. G. (2004). Notch activation regulates the segregation and differentiation of rhombomere boundary cells in the zebrafish hindbrain. Dev. Cell 6, 539-550.

Cho, K. O., and Choi, K. W. (1998). Fringe is essential for mirror symmetry and morphogenesis in the Drosophila eye. Nature 396, 272-276.

Concha, M. L., and Wilson, S. W. (2001). Asymmetry in the epithalamus of vertebrates. J. Anat. 199, 63-84.

Crossley, P. H., Martinez, S., Ohkubo, Y., and Rubenstein, J. L. (2001). Coordinate expression of Fgf8, Otx2, Bmp4, and Shh in the rostral prosencephalon during development of the telencephalic and optic vesicles. Neuroscience 108, 183-206.

Dominguez, M., and de Celis, J. F. (1998). A dorsal/ventral boundary established by notch controls growth and polarity in the Drosophila eye. Nature 396, 276-278.

Echevarria, D., Vieira, C., Gimeno, L., and Martinez, S. (2003). Neuroepithelial secondary organizers and cell fate specification in the developing brain. Brain Res. Brain Res. Rev. 43 , 179-191.

Figdor, M. C., and Stern, C. D. (1993). Segmental organization of embryonic diencephalon. Nature 363, 630-634.

Fishell, G., Mason, C. A., and Hatten, M. E. (1993). Dispersion of neural progenitors within the germinal zones of the forebrain. Nature 362, 636-638.

Fraser, S., Keynes, R., and Lumsden, A. (1990). Segmentation in the chick embryo hindbrain is defined by cell lineage restrictions. Nature 344, 431-435.

Gamse, J. T., Kuan, Y. S., Macurak, M., Brosamle, C., Thisse, B., Thisse, C., and Halpern, M. E. (2005). Directional asymmetry of the zebrafish epithalamus guides dorsoventral innervation of the midbrain target. Development 132, 4869-4881.

Garcia-Lopez, R., Vieira, C., Echevarria, D., and Martinez, S. (2004). Fate map of the diencephalon and the zona limitans at the 10-somites stage in chick embryos. Dev. Biol. 268, 514-530.

Giger, R. J., Cloutier, J. F., Sahay, A., Prinjha, R. K., Levengood, D. V., Moore, S. E., Pickering, S., Simmons, D., Rastan, S., Walsh, F. S., Kolodkin, A. L., Ginty, D. D., and Geppert, M. (2000). Neuropilin-2 is required in vivo for selective axon guidance responses to secreted semaphorins. Neuron 25, 29-41. 
Gimeno, L., and Martinez, S. (2007). Expression of chick Fgf19 and mouse Fgf15 orthologs is regulated in the developing brain by Fgf8 and Shh. Dev. Dyn. 236, 2285-2297.

Golden, J. A., and Cepko, C. L. (1996). Clones in the chick diencephalon contain multiple cell types and siblings are widely dispersed. Development 122, 65-78.

Gomez-Skarmeta, J. L., and Modolell, J. (2002). Iroquois genes: genomic organization and function in vertebrate neural development. Curr. Opin. Genet. Dev. 12, 403-408.

Guinazu, M. F., Chambers, D., Lumsden, A., and Kiecker, C. (2007). Tissue interactions in the developing chick diencephalon. Neural Dev. 2, 25.

Halpern, M. E., Liang, J. O., and Gamse, J. T. (2003). Leaning to the left: laterality in the zebrafish forebrain. Trends Neurosci. 26, 308-313.

Hashimoto-Torii, K., Motoyama, J., Hui, C. C., Kuroiwa, A., Nakafuku, M., and Shimamura, K. (2003). Differential activities of Sonic hedgehog mediated by Gli transcription factors define distinct neuronal subtypes in the dorsal thalamus. Mech. Dev. 120, 1097-1111.

Heisenberg, C. P., Houart, C., TakeUchi, M., Rauch, G. J., Young, N., Coutinho, P., Masai, I., Caneparo, L., Concha, M. L., Geisler, R., Dale, T. C., Wilson, S. W., and Stemple, D. L. (2001). A mutation in the Gsk3binding domain of zebrafish Masterblind/Axin1 leads to a fate transformation of telencephalon and eyes to diencephalon. Genes Dev. 15, 1427-1434.

Hevner, R. F., Miyashita-Lin, E., and Rubenstein, J. L. (2002). Cortical and thalamic axon pathfinding defects in Tbr1, Gbx2, and Pax6 mutant mice: evidence that cortical and thalamic axons interact and guide each other. J. Comp. Neurol. 447, 8-17.

Hikosaka, O. (2010). The habenula: from stress evasion to value-based decision-making. Nat. Rev. Neurosci. 11, 503-513.

Hikosaka, O., Sesack, S. R., Lecourtier, L., and Shepard, P. D. (2008). Habenula: crossroad between the basal ganglia and the limbic system. J. Neurosci. 28, 11825-11829.

Hirata, T., Nakazawa, M., Muraoka, O., Nakayama, R., Suda, Y., and Hibi, M. (2006). Zinc-finger genes $\mathrm{Fez}$ and Fez-like function in the establishment of diencephalon subdivisions. Development 133, 3993-4004.
Hirata, T., Suda, Y., Nakao, K., Narimatsu, M., Hirano, T., and Hibi, M. (2004). Zinc finger gene fezlike functions in the formation of subplate neurons and thalamocortical axons. Dev. Dyn. 230, 546-556.

Houart, C., Caneparo, L., Heisenberg, C., Barth, K., Take-Uchi, M., and Wilson, S. (2002). Establishment of the telencephalon during gastrulation by local antagonism of Wnt signaling. Neuron 35, 255-265.

Houweling, A. C., Dildrop, R., Peters, T., Mummenhoff, J., Moorman, A. F., Ruther, U., and Christoffels, V. M. (2001). Gene and cluster-specific expression of the Iroquois family members during mouse development. Mech. Dev. 107, 169-174.

Ingram, W. J., Mccue, K. I., Tran, T. H., Hallahan, A. R., and Wainwright, B. J. (2008). Sonic hedgehog regulates Hes1 through a novel mechanism that is independent of canonical notch pathway signalling. Oncogene 27, 1489-1500.

Irvine, K. D., and Rauskolb, C. (2001). Boundaries in development: formation and function. Annu. Rev. Cell Dev. Biol. 17, 189-214.

Ishibashi, M., and McMahon, A. P. (2002). A sonic hedgehogdependent signaling relay regulates growth of diencephalic and mesencephalic primordia in the early mouse embryo. Development 129, 4807-4819.

Jeong, J. Y., Einhorn, Z., Mathur, P., Chen, L., Lee, S., Kawakami, K., and Guo, S. (2007). Patterning the zebrafish diencephalon by the conserved zinc-finger protein Fezl. Development 134, 127-136.

Jeong, Y., Dolson, D. K., Waclaw, R. R., Matise, M. P., Sussel, L., Campbell, K., Kaestner, K. H., and Epstein, D. J. (2011). Spatial and temporal requirements for Sonic hedgehog in the regulation of thalamic interneuron identity. Development 138, 531-541.

Jones, E. G. (2007). The thalamus. Cambridge, NY: Cambridge University Press.

Jukkola, T., Lahti, L., Naserke, T., Wurst, W., and Partanen, J. (2006). FGF regulated gene-expression and neuronal differentiation in the developing midbrain-hindbrain region. Dev. Biol. 297, 141-157.

Kaellen, B. (1965). Degeneration and regeneration in the vertebrate central nervous system during embryogenesis. Prog. Brain Res. 14, 77-96.

Kageyama, R., Ohtsuka, T., and Kobayashi, T. (2008). Roles of
Hes genes in neural development. Dev. Growth Differ. 50(Suppl. 1) S97-S103.

Kantor, D. B., Chivatakarn, O., Peer, K. L., Oster, S. F., Inatani, M., Hansen, M. J., Flanagan, J. G., Yamaguchi, Y., Sretavan, D. W., Giger, R. J., and Kolodkin, A. L. (2004). Semaphorin $5 \mathrm{~A}$ is a bifunctional axon guidance cue regulated by heparan and chondroitin sulfate proteoglycans. Neuron 44, 961-975.

Kataoka, A., and Shimogori, T. (2008). Fgf8 controls regional identity in the developing thalamus. Development 135, 2873-2881.

Kiecker, C., and Lumsden, A. (2004). Hedgehog signaling from the ZLI regulates diencephalic regional identity. Nat. Neurosci. 7, 1242-1249.

Kiecker, C., and Lumsden, A. (2005). Compartments and their boundaries in vertebrate brain development. Nat. Rev. Neurosci. 6, 553-564.

Kobayashi, D., Kobayashi, M., Matsumoto, K., Ogura, T., Nakafuku, M., and Shimamura, K. (2002). Early subdivisions in the neural plate define distinct competence for inductive signals. Development 129 , 83-93.

Kurokawa, D., Kiyonari, H., Nakayama, R., Kimura-Yoshida, C., Matsuo, I., and Aizawa, S. (2004). Regulation of Otx2 expression and its functions in mouse forebrain and midbrain. Development 131, 3319-3331.

Langenberg, T., and Brand, M. (2005). Lineage restriction maintains a stable organizer cell population at the zebrafish midbrain-hindbrain boundary. Development 132, 3209-3216.

Larsen, C. W., Zeltser, L. M., and Lumsden, A. (2001). Boundary formation and compartition in the avian diencephalon. J. Neurosci. 21, 4699-4711.

Lavado, A., Lagutin, O. V., and Oliver, G. (2008). Six3 inactivation causes progressive caudalization and aberrant patterning of the mammalian diencephalon. Development 135 441-450.

Libby, R. T., Smith, R. S., Savinova, O. V., Zabaleta, A., Martin, J. E., Gonzalez, F. J., and John, S. W. (2003). Modification of ocular defects in mouse developmental glaucoma models by tyrosinase. Science 299, 1578-1581.

Liu, C., Wang, Y., Smallwood, P. M. and Nathans, J. (2008). An essential role for Frizzled5 in neuronal survival in the parafascicular nucleus of the thalamus. J. Neurosci. 28, 5641-5653.

Lumsden, A., and Krumlauf, R. (1996). Patterning the vertebrate neuraxis. Science 274, 1109-1115.
Martinez-Ferre, A., and Martinez, S. (2009). The development of the thalamic motor learning area is regulated by Fgf8 expression. J. Neurosci. 29, 13389-13400.

Mattes, B., Weber, S., Peres, J., Chen, Q., Davidson, G., Houart, C., and Scholpp, S. (2012). Wnt3 and Wnt3a are required for induction of the mid-diencephalic organizer in the caudal forebrain. Neural Dev. 7, 12.

Meinhardt, H. (1983). Cell determination boundaries as organizing regions for secondary embryonic fields. Dev. Biol. 96, 375-385.

Miyake, A., Nakayama, Y., Konishi, M., and Itoh, N. (2005). Fgf19 regulated by $\mathrm{Hh}$ signaling is required for zebrafish forebrain development. Dev. Biol. 288, 259-275.

Miyashita-Lin, E. M., Hevner, R., Wassarman, K. M., Martinez, S., and Rubenstein, J. L. (1999). Early neocortical regionalization in the absence of thalamic innervation. Science 285, 906-909.

Morris, J. S., Smith, K. A., Cowen, P. J., Friston, K. J., and Dolan, R. J. (1999). Covariation of activity in habenula and dorsal raphe nuclei following tryptophan depletion. Neuroimage 10, 163-172.

Nakagawa, Y., and O'Leary, D. D. (2001). Combinatorial expression patterns of LIM-homeodomain and other regulatory genes parcellate developing thalamus. J. Neurosci. 21, 2711-2725.

Papayannopoulos, V., Tomlinson, A., Panin, V. M., Rauskolb, C., and Irvine, K. D. (1998). Dorsal-ventral signaling in the Drosophila eye. Science 281, 2031-2034.

Peters, T., Ausmeier, K., Dildrop, R., and Ruther, U. (2002). The mouse Fused toes $(\mathrm{Ft})$ mutation is the result of a 1.6-Mb deletion including the entire Iroquois B gene cluster. Mamm. Genome 13, 186-188.

Peters, T., Dildrop, R., Ausmeier, K., and Ruther, U. (2000). Organization of mouse Iroquois homeobox genes in two clusters suggests a conserved regulation and function in vertebrate development. Genome Res. 10, 1453-1462.

Peukert, D., Weber, S., Lumsden, A., and Scholpp, S. (2011). Lhx2 and lhx 9 determine neuronal differentiation and compartition in the caudal forebrain by regulating wnt signaling. PLoS Biol. 9, e1001218. doi:10.1371/journal.pbio.1001218

Puelles, E., Acampora, D., Gogoi, R., Tuorto, F., Papalia, A., Guillemot, F., Ang, S. L., and Simeone, A. (2006). Otx2 controls identity and fate of glutamatergic progenitors of 
the thalamus by repressing GABAergic differentiation. J. Neurosci. 26, 5955-5964.

Puelles, L., Amat, J. A., and MartinezDe-La-Torre, M. (1987). Segmentrelated, mosaic neurogenetic pattern in the forebrain and mesencephalon of early chick embryos: I. Topography of AChE-positive neuroblasts up to stage HH18. J. Comp. Neurol. 266, 247-268.

Puelles, L., and Rubenstein, J. L. (1993). Expression patterns of homeobox and other putative regulatory genes in the embryonic mouse forebrain suggest a neuromeric organization. Trends Neurosci. 16, 472-479.

Puelles, L., and Rubenstein, J. L. (2003). Forebrain gene expression domains and the evolving prosomeric model. Trends Neurosci. 26, 469-476.

Quina, L. A., Wang, S., Ng, L., and Turner, E. E. (2009). Brn3a and Nurr1 mediate a gene regulatory pathway for habenula development. J. Neurosci. 29, 14309-14322.

Quinlan, R., Graf, M., Mason, I., Lumsden, A., and Kiecker, C. (2009). Complex and dynamic patterns of Wnt pathway gene expression in the developing chick forebrain. Neural Dev. 4,35 .

Rauskolb, C., Correia, T., and Irvine, K. D. (1999). Fringe-dependent separation of dorsal and ventral cells in the Drosophila wing. Nature 401, 476-480.

Redies, C., Ast, M., Nakagawa, S., Takeichi, M., Martinez-De-La-Torre, M., and Puelles, L. (2000). Morphologic fate of diencephalic prosomeres and their subdivisions revealed by mapping cadherin expression. J. Comp. Neurol. 421, 481-514.

Redies, C., and Takeichi, M. (1996). Cadherins in the developing central nervous system: an adhesive code for segmental and functional subdivisions. Dev. Biol. 180, 413-423.

Rodriguez-Seguel, E., Alarcon, P., and Gomez-Skarmeta, J. L. (2009). The Xenopus Irx genes are essential for neural patterning and define the border between prethalamus and thalamus through mutual antagonism with the anterior repressors Fezf and Arx. Dev. Biol. 329, 258-268.

Rose, J. E. (1949). The ontogenetic development of the rabbit's diencephalon. J. Comp. Neurol. 77, 61-129.

Rubenstein, J. L., Martinez, S., Shimamura, K., and Puelles, L. (1994). The embryonic vertebrate forebrain: the prosomeric model. Science 266, 578-580.
Sakurai, Y., Kurokawa, D., Kiyonari, H., Kajikawa, E., Suda, Y., and Aizawa, S. (2010). Otx2 and Otx1 protect diencephalon and mesencephalon from caudalization into metencephalon during early brain regionalization. Dev. Biol. 347, 392-403.

Sato, T., Joyner, A. L., and Nakamura, H. (2004). How does Fgf signaling from the isthmic organizer induce midbrain and cerebellum development? Dev. Growth Differ. 46, 487-494.

Sato, T., Shimazaki, T., Naka, H., Fukami, S., Satoh, Y., Okano, H., Lax, I., Schlessinger, J., and Gotoh, N. (2010). FRS2alpha regulates Erk levels to control a self-renewal target Hes1 and proliferation of FGFresponsive neural stem/progenitor cells. Stem Cells 28, 1661-1673.

Scholpp, S., Delogu, A., Gilthorpe, J., Peukert, D., Schindler, S., and Lumsden, A. (2009). Her6 regulates the neurogenetic gradient and neuronal identity in the thalamus. Proc. Natl. Acad. Sci. U.S.A. 106, 19895-19900.

Scholpp, S., Foucher, I., Staudt, N., Peukert, D., Lumsden, A., and Houart, C. (2007). Otx1l, Otx2 and Irxlb establish and position the ZLI in the diencephalon. Development 134, 3167-3176.

Scholpp, S., and Lumsden, A. (2010). Building a bridal chamber: development of the thalamus. Trends Neurosci. 33, 373-380.

Scholpp, S., Wolf, O., Brand, M., and Lumsden, A. (2006). Hedgehog signalling from the zona limitans intrathalamica orchestrates patterning of the zebrafish diencephalon. Development 133, 855-864.

Simeone, A., Gulisano, M., Acampora, D., Stornaiuolo, A., Rambaldi, M., and Boncinelli, E. (1992). Two vertebrate homeobox genes related to the Drosophila empty spiracles gene are expressed in the embryonic cerebral cortex. EMBO J. 11, 2541-2550.

Staudt, N., and Houart, C. (2007). The prethalamus is established during gastrulation and influences diencephalic regionalization. PLoS Biol. 5, e69. doi:10.1371/journal.pbio.0050069

Sunmonu, N. A., Li, K., Guo, Q., and Li, J. Y. (2011). Gbx2 and Fgf8 are sequentially required for formation of the midbrain-hindbrain compartment boundary. Development 138, 725-734.

Sutherland, R. J. (1982). The dorsal diencephalic conduction system: a review of the anatomy and functions of the habenular complex. Neurosci. Biobehav. Rev. 6, 1-13.

Sylvester, J. B., Rich, C. A., Loh, Y. H., Van Staaden, M. J., Fraser, G. J., and
Streelman, J. T. (2010). Brain diversity evolves via differences in patterning. Proc. Natl. Acad. Sci. U.S.A. 107, 9718-9723.

Szabo, N. E., Zhao, T., Zhou, X., and Alvarez-Bolado, G. (2009). The role of Sonic hedgehog of neural origin in thalamic differentiation in the mouse. J. Neurosci. 29, 2453-2466.

Tossell, K., Andreae, L. C., Cudmore, C., Lang, E., Muthukrishnan, U., Lumsden, A., Gilthorpe, J. D., and Irving, C. (2011a). Lrrn1 is required for formation of the midbrainhindbrain boundary and organiser through regulation of affinity differences between midbrain and hindbrain cells in chick. Dev. Biol. 352, 341-352.

Tossell, K., Kiecker, C., Wizenmann, A., Lang, E., and Irving, C. (2011b). Notch signalling stabilises boundary formation at the midbrainhindbrain organiser. Development 138, 3745-3757.

Ullsperger, M., and von Cramon, D. Y. (2003). Error monitoring using external feedback: specific roles of the habenular complex, the reward system, and the cingulate motor area revealed by functional magnetic resonance imaging. J. Neurosci. 23 , 4308-4314.

Vaage, S. (1969). The segmentation of the primitive neural tube in chick embryos (Gallus domesticus). A morphological, histochemical and autoradiographical investigation. Ergeb. Anat. Entwicklungsgesch. 41, 3-87.

Vieira, C., Garda, A. L., Shimamura, K., and Martinez, S. (2005). Thalamic development induced by Shh in the chick embryo. Dev. Biol. 284, 351-363.

Vue, T. Y., Aaker, J., Taniguchi, A., Kazemzadeh, C., Skidmore, J. M., Martin, D. M., Martin, J. F., Treier, M., and Nakagawa, Y. (2007). Characterization of progenitor domains in the developing mouse thalamus. J. Comp. Neurol. 505, 73-91.

Vue, T. Y., Bluske, K., Alishahi, A., Yang, L. L., Koyano-Nakagawa, N., Novitch, B., and Nakagawa, Y. (2009). Sonic hedgehog signaling controls thalamic progenitor identity and nuclei specification in mice. J. Neurosci. 29, 4484-4497.

Wall, D. S., Mears, A. J., Mcneill, B., Mazerolle, C., Thurig, S., Wang, Y., Kageyama, R., and Wallace, V. A. (2009). Progenitor cell proliferation in the retina is dependent on Notchindependent Sonic hedgehog/Hes1 activity. J. Cell Biol. 184, 101-112.

Walshe, J., and Mason, I. (2003). Unique and combinatorial functions of Fgf3 and Fgf8 during zebrafish forebrain development. Development 130, 4337-4349.

Wang, Y., Thekdi, N., Smallwood, P. M., Macke, J. P., and Nathans, J. (2002). Frizzled-3 is required for the development of major fiber tracts in the rostral CNS. J. Neurosci. 22 , 8563-8573.

Wingate, R. J., and Lumsden, A. (1996). Persistence of rhombomeric organisation in the postsegmental hindbrain. Development 122, 2143-2152.

Wurst, W., and Bally-Cuif, L. (2001). Neural plate patterning: upstream and downstream of the isthmic organizer. Nat. Rev. Neurosci. 2 99-108.

Xiang, M., Gan, L., Zhou, L., Klein, W. H., and Nathans, J. (1996). Targeted deletion of the mouse POU domain gene Brn-3a causes selective loss of neurons in the brainstem and trigeminal ganglion, uncoordinated limb movement, and impaired suckling. Proc. Natl. Acad. Sci. U.S.A. 93, 11950-11955.

Yoon, M. S., Puelles, L., and Redies, C. (2000). Formation of cadherinexpressing brain nuclei in diencephalic alar plate divisions. J. Comp. Neurol. 427, 461-480.

Zeltser, L. M. (2005). Shh-dependent formation of the ZLI is opposed by signals from the dorsal diencephalon. Development 132, 2023-2033.

Zeltser, L. M., Larsen, C. W., and Lumsden, A. (2001). A new developmental compartment in the forebrain regulated by lunatic fringe. Nat. Neurosci. 4, 683-684.

Zervas, M., Millet, S., Ahn, S., and Joyner, A. L. (2004). Cell behaviors and genetic lineages of the mesencephalon and rhombomere 1. Neuron 43, 345-357.

Zhang, N., and Gridley, T. (1998). Defects in somite formation in lunatic fringe-deficient mice. Nature 394, 374-377.

Zhou, C. J., Pinson, K. I., and Pleasure, S. J. (2004). Severe defects in dorsal thalamic development in lowdensity lipoprotein receptor-related protein-6 mutants. J. Neurosci. 24, 7632-7639.

Zhou, L., Bar, I., Achouri, Y., Campbell, K., De Backer, O., Hebert, J. M., Jones, K., Kessaris, N., De Rouvroit, C. L., O'leary, D., Richardson, W. D., Goffinet, A. M., and Tissir, F. (2008). Early forebrain wiring: genetic dissection using conditional Celsr3 mutant mice. Science 320, 946-949.

Zhou, L., Qu, Y., Tissir, F., and Goffinet, A. M. (2009). Role ofthe 
atypical cadherin Celsr3 during development of the internal capsule. Cereb. Cortex 19(Suppl. 1), i114-i119.

Conflict of Interest Statement: The authors declare that the research was conducted in the absence of any commercial or financial relationships that could be construed as a potential conflict of interest.

Received: 01 February 2012; paper pending published: 12 March 2012; accepted: 17 April 2012; published online: 11 May 2012.
Citation: Chatterjee $M$ and Li JYH (2012) Patterning and compartment formation in the diencephalon. Front. Neurosci. 6:66. doi: 10.3389/fnins.2012.00066

This article was submitted to Frontiers in Neurogenesis, a specialty of Frontiers in Neuroscience.
Copyright $($ C 2012 Chatterjee and Li. This is an open-access article distributed under the terms of the Creative Commons Attribution Non Commercial License, which permits non-commercial use, distribution, and reproduction in other forums, provided the original authors and source are credited. 